\title{
A CHARACTERIZATION OF TOTAL GRAPHS
}

\author{
M. BEHZAD
}

I. ABStRact. We consider "ordinary" graphs; that is, finite undirected graphs with no loops or multiple edges. The total graph $T(G)$ of a graph $G$ is that graph whose vertex set is $V(G) \cup E(G)$ and in which two vertices are adjacent if and only if they are adjacent or incident in $G$. A characterization of regular total graphs as well as some other properties of total graphs have been considered before. In this article we consider nonregular graphs and yield a method which enables us actually to determine whether or not they are total.

II. Introduction. We consider (ordinary) graphs; that is, finite undirected graphs with no loops or multiple edges. Besides the chromatic number $\chi(G)$ and edge chromatic number $\chi_{1}(G)$ there is associated with $G$ another positive integer $\chi_{2}(G)$, called the total chromatic number of $G$, which is the minimum number of colors required for coloring the elements (edges and vertices) of $G$ such that no two elements which are either adjacent or incident have the same color. (The Total Chromatic Conjecture [3] states that $\chi_{2}(G) \leqq 2$ $+\max \operatorname{deg} G$. In this conjecture $G$ can be replaced by multigraphs $M$ containing no 4-regular multigraph of order 3 as a subgraph. ${ }^{1}$ ) The total graph $T(G)$ of $G$ is defined in such a way that $\chi_{2}(G)=\chi(T(G))$-in analogy with the well-known formula $\chi_{1}(G)$ $=\chi(L(G))$, where $L(G)$ is the line graph of $G$. The total graph $T(G)$ of $G$ is that graph whose vertex set is $V(G) \cup E(G)$, and in which two vertices are adjacent if and only if they are adjacent or incident in $G$. For an illustration a graph $G$ is given in Figure 1 together with $L(G)$ and $T(G)$. Both $G$ and $L(G)$ are disjoint induced subgraphs of $T(G)$.

A characterization of regular total graphs is given in [7] and some other properties of total graphs are considered in [1], [2], [4]. In this article we yield a method which enables us actually to determine whether or not any given graph is total.

III. Results. In [6] it was proved that $G_{1}=G_{2}$-isomorphism is denoted by the equality sign-if and only if $T\left(G_{1}\right)=T\left(G_{2}\right)$. There, it

Received by the editors January 25, 1970.

AMS 1969 subject classifications. Primary 0540.

Key words and phrases. Total graphs.

1 This restriction on $M$ is necessary as was observed by the author, E. Jacovix and others. 


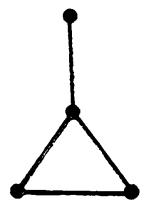

$\boldsymbol{G}$

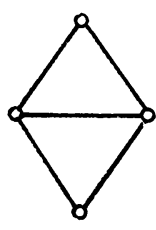

$L(G)$

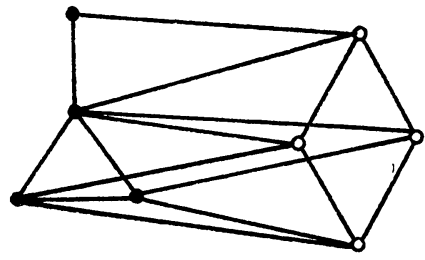

$T(G)$

FIGURE 1

was also shown that, apart from isomorphism, $G$ is the only subgraph of $H=T(G)$ whose total graph is $H$, where $G$ is a connected graph which is neither a cycle $C$ nor a complete graph $K$. (Definitions not given here may be found in [5], [8].) This subgraph called the special subgraph of $H$ is denoted by $G_{s}$. The subgraph $G_{8}$ is induced by a set of vertices of $H$ which are called the special vertices of $H$; any other element of $V(H)$ is a nonspecial element of $V(H)$. The subgraph induced by the set of nonspecial vertices of $H$ is $L\left(G_{s}\right)$ and in $H$ each nonspecial vertex is adjacent with exactly two adjacent special elements.

Suppose that $H$ is a connected total graph which is neither $T(C)$ nor $T(K)$, and that $v \in V(H)$ is nonspecial. Let $\{i\}, i=0,1,2, \cdots$, $n$, denote the class of all vertices of $H$ whose distance from $v$ is $i$. Our hypotheses imply that $n \geqq 2$. Then we have the following theorem.

TheOREM 1. Let $H, H \neq T(C), T(K)$, be a connected total graph and let $v$ be a nonspecial element of $V(H)$. Then each nonspecial vertex of $H$ in $\{i\}, i \geqq 1$, is adjacent with exactly two special vertices of $H$ both of which are in $\{i\}$, or one is in $\{i\}$ and the other in $\{i+1\}$.

Proof. We use induction on $i$. Let the two adjacent special vertices of $H$ which are adjacent with $v$ be denoted by $u_{1}$ and $v_{1}$. It is clear that $u_{1}$ and $v_{1}$ are the only special elements of $V(H)$ which are in $\{1\}$; and that each nonspecial element of $\{1\}$ is adjacent with two special vertices of $H$ one of which is in $\{2\}$ and the other in $\{1\}$.

Let $w$ be a nonspecial vertex of $H$ in $\{2\}$. If $w$ is adjacent with $u_{1}$ or $v_{1}$, then $w$ and $v$ are adjacent in $H$ and $w \in\{1\}$ which is a contradiction. Clearly $w$ is adjacent with no element of $\{i\}, i \geqq 4$. Next, we show that $w$ is not adjacent with two special elements of $\{3\}$. Assume this is the case. Then the vertex $w$, which is adjacent neither with $u_{1}$ nor with $v_{1}$, is adjacent with a nonspecial element $w_{1}$ of $\{1\}$. But this is impossible, since the preimages of $w$ and $w_{1}$ under the total graph function have no vertices in common. Hence the assertion follows for $\{2\}$. 


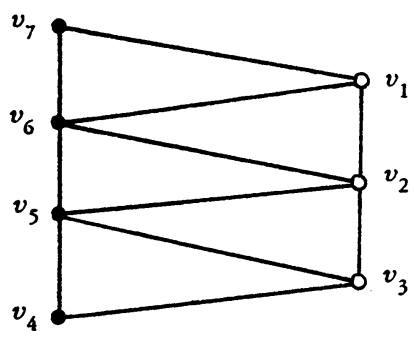

FIGURE 2

Assume the assertion is true for $i, 2 \leqq i<n$, and let $w$ be a nonspecial vertex of $H$ in $\{i+1\}$. First, we show that $w$ is not adjacent with two special elements of $\{i+2\}$. Assume this is the case. The vertex $w$ is adjacent with an element $w_{i}$ of $\{i\}$. The vertex $w_{i}$ is not special since otherwise $w$ is adjacent with three or more special vertices of $H . w_{1}$ is not nonspecial either, because otherwise the induction hypothesis would imply that the vertices $w$ and $w_{i}$ are not adjacent.

Next, we show that $w$ is not adjacent with any special vertex of $H$ in $\{i\}$. Assume this is not the case, and let $u_{1}$ be a special vertex of $H$ adjacent with $w$ which is in $\{i\}$. The vertex $u_{i}$ is adjacent with a vertex $u$ of $\{i-1\}$. If $u$ is special, then by the induction hypothesis the image of the edge $u u_{i}$ under the total graph function, say $u_{i-1}$, is in $\{i-1\}$, and the vertices $w$ and $u_{i-1}$ are adjacent. Thus $w \notin\{i+1\}$ which contradicts our assumption. Hence $u$ is a nonspecial vertex of $\{i-1\}$. Then $u$ is adjacent with a special element of $\{i-1\}$, say $u^{\prime}$. In this case the image of the edge $u_{i} u^{\prime}$ is $u$. Hence $u$ and $w$ are adjacent and it follows that $w \notin\{i+1\}$. This contradiction proves our assertion and completes the proof of the theorem.

The following corollaries are of value to us.

COROLlaRY 1. Under the assumptions of Theorem 1, no nonspecial element of $\{i\}$ is adjacent with a special element of $\{i-1\}, i \geqq 2$.

CoROLlaRy 2. Under the assumptions of Theorem 1, every class $\{i\}, 1$ $\leqq i \leqq n-1$, contains both special and nonspecial vertices of $H$; while the class $\{n\}$ cannot solely contain some nonspecial vertices of $H$.

Proof. Assume $H=T(G)$. Then $G$ and $L(G)$ are two disjoint connected subgraphs of $H$. Hence if $\{i\}, 1 \leqq i \leqq n-1$, solely contains nonspecial (resp. special) vertices of $H$, then the class $\{j\}, j \geqq i+1$, cannot contain any special (resp. nonspecial) element of $V(H)$. This observation together with Theorem 1 imply that no class $\{i\}, 1$ 
$\leqq i \leqq n$, can entirely contain some nonspecial vertices of $H$. Next, we show that no class $\{i\}, 1 \leqq i \leqq n-1$, can contain only special elements. Assume so, and let $u \in\{i\}$ for which there exists a special element $w$ in $\{i+1\}$ adjacent with $u$. Then, under the total graph function the edge $u w$ has no image. This contradiction proves the corollary.

In Figure 2 we present a graph $H$ which is total and in which the class $\{n\}=\{3\}$ contains a special element alone. (We note that $G_{8}$ is the path $v_{4}, v_{5}, v_{6}, v_{7}$. Hence the vertices $v_{1}, v_{2}$ and $v_{3}$ are the nonspecial vertices of $H$.) The classes $\{i\}, i=0,1,2,3$, are determined with respect to the vertex $v_{1}$ and they are: $\{0\}=\left\{v_{1}\right\},\{1\}=\left\{v_{2}, v_{6}, v_{7}\right\}$, $\{2\}=\left\{v_{3}, v_{5}\right\}$, and $\{3\}=\left\{v_{4}\right\}$.

COROLlaRY 3. Under the assumptions of Theorem 1, each nonspecial element in $\{i\}$ is adjacent with at least one nonspecial element in $\{i-1\}$, for $i \geqq 1$, and each special element in $\{i\}, i \geqq 2$, is adjacent with at least one special element in $\{i-1\}$.

Proof. This follows directly from Theorem 1 , and the fact that both $G_{s}$ and $L\left(G_{s}\right)$ are two connected subgraphs of $H$.

Theorem 2. Assume $H, H \neq T(C), T(K)$, is a connected total graph and that $v$ is a nonspecial element of $V(H)$ adjacent with the special vertices $v_{1}$ and $u_{1}$ of $H$. Then we can determine the subgraph $G_{s}$ completely.

Proof. Since $G_{s}$ is an induced subgraph of $H$, it suffices to determine the set of special vertices of $H$. We do this by separating the sets of special and the sets of nonspecial elements $S_{i}$ and $N_{i}$, respectively, of each class $\{i\}, i=0,1,2, \cdots, n$, formed with respect to the vertex $v$.

It is clear that the class $\{0\}$ contains no special element. Thus $S_{0}=\varnothing$, and $N_{0}=\{0\}-S_{0}=\{0\}=\{v\}$. The class $\{1\}$ contains exactly two special elements and they are the vertices $u_{1}$ and $v_{1}$. Therefore $S_{1}=\left\{u_{1}, v_{1}\right\}$, and $N_{1}=\{1\}-S_{1}$. Clearly $N_{1} \neq \varnothing$.

Let $w_{1} \in N_{1}$. Then by the proof of Theorem $1, w_{1}$ is adjacent with one of $u_{1}$ and $v_{1}$, say $u_{1}$, and a special element of $H$, say $u_{2}$, which is in $\{2\}$. This vertex $u_{2}$ which we propose to determine is necessarily adjacent with $u_{1}$. Since no nonspecial element of $\{2\}$ is adjacent with a special element of $\{1\}$ any element of $\{2\}$ adjacent with $u_{1}$ is necessarily special. Among these only one is adjacent with both $u_{1}$ and $w_{1}$ since otherwise the vertex $w_{1}$ will be adjacent with three or more special vertices of $H$. Thus the vertex $u_{2}$ can uniquely be determined. We repeat this argument for all elements of $N_{1}$ and in this manner 
we obtain a set $S_{2}^{\prime}$ consisting of some special elements of $\{2\}$. We observe that $S_{2}^{\prime} \neq \varnothing$ since $N_{1} \neq \varnothing$. Let $w_{2} \in\{2\}-S_{2}^{\prime}$. The vertex $w_{2}$ is a nonspecial element of $H$. For otherwise, by Corollary 3 , the vertex $w_{2}$ is adjacent with a special element, say $v_{1}^{\prime}$, of $\{1\}$ and the edge $w_{2} v_{1}^{\prime}$ must correspond, under the total graph function, to a nonspecial element, say $w_{1}^{\prime}$, of $H$. But the vertex $w_{1}^{\prime}$ can only be in the class $\{1\}$. This contradicts the fact that $w_{2}$ is an element of $\{2\}-S_{2}^{\prime}$. Thus $N_{2}=\{2\}-S_{2}^{\prime}$ and $S_{2}=S_{2}^{\prime}$.

Next, we separate special and nonspecial elements of $\{3\}$. Let $w_{2} \in N_{2}$. If $w_{2}$ is adjacent with two elements of $S_{2}$, then each element of $\{3\}$ adjacent with $w_{2}$ is a nonspecial element of $\{3\}$; otherwise $w_{2}$ is adjacent with a special element, say $u_{2}$, of $\{2\}$ and a special element, say $u_{3}$, of $\{3\}$. We propose to determine $u_{3}$. Again, as was seen before, all vertices of $\{3\}$ adjacent with $u_{2}$ are special vertices of $\{3\}$ among which only one is adjacent with both $w_{2}$ and $u_{2}$. This vertex is the vertex $u_{3}$ which we are looking for. We repeat this argument for every element of $N_{2}$ and obtain a set $S_{3}^{\prime}$ consisting of some special elements of $\{3\}$. We observe that every element of $\{3\}-S_{3}^{\prime}$ is nonspecial. (The proof is similar to the one given for $\{2\}-S_{2}^{\prime}$.) Thus $N_{3}=\{3\}-S_{3}^{\prime}$, and $S_{3}=S_{3}^{\prime}$.

Using induction and the above procedure we separate the special and nonspecial elements of $\{i\}, i=0,1,2, \cdots, n$. Now we let $S=S_{0} \cup S_{1} \cup \ldots \cup S_{n}$. Then $G_{s}=\langle S\rangle$, and $H=T\left(G_{s}\right)$. This completes the proof of the theorem.

Since in the above theorem the vertices $v, u_{1}$, and $v_{1}$ play an important role for the determination of $G_{s}$, we denote $G_{s}$ by $G_{i ; u_{1} v_{1}}$.

Let $u$ be an arbitrary vertex of a graph $H$. We denote the set consisting of $u$ and all vertices of $H$ adjacent with $u$ by $\bar{N}(u)$; this set is called the closed neighborhood of $u$. Now we are prepared to present the main theorem of this article.

Theorem 3. Assume that $H, H \neq T(C), T(K)$, is a connected graph, and that $u$ is an arbitrary vertex of $H$. Then $H$ is total if and only if $H$ $=T\left(G_{v ; u_{1} v_{1}}\right)$ for some $v \in \bar{N}(u)$ and some edge $u_{1} v_{1}$, where $u_{1}$ and $v_{1}$ are two even vertices of $H$ adjacent with $v$.

Proof. Assume that $H$ is total. Then some element $v$ of $\bar{N}(u)$ is a nonspecial vertex of $H$. Under the total graph function, $v$ corresponds to an edge $u_{1} v_{1}$ of the special subgraph of $H$. The vertices $u_{1}$ and $v_{1}$ have even degrees in $H$ and by the definition of total graphs both $u_{1}$ and $v_{1}$ are adjacent with $v$. Thus the special subgraph of $H$ is $G_{v ; u_{1} v_{1}}$ and $H=T\left(G_{v ; u_{1} v_{1}}\right)$. The converse is trivial.

Theorems 2 and 3 answer the main question mentioned at the end 
of the introduction, namely, when is $H$ total. The answer is that we try appropriate $v, u_{1}, v_{1}$, find $G_{v ; u_{1} v_{1}}$ by the algorithm provided in the proof of Theorem 2, and then see if $T\left(G_{v ; u_{1} v_{1}}\right)$ is $H$.

The characterization of disconnected total graphs is reduced to that of connected ones since if $G$ has $m$ components, then $T(G)$ consists of $m$ components each of which is the total graph of a component of $G$. The converse is also true. Thus to completely characterize total graphs it remains to give a characterization of $T(C)$ and $T(K)$. The following results are obtained in [7] and for completeness we state them next.

(i) Let $G$ be a connected regular graph of degree 4 . The only such graph with fewer than seven vertices is $T\left(K_{3}\right)$ which is the total graph of each of its triangles. If $|V(G)| \geqq 7$, then $G$ is total if and only if: (a) $|V(G)|=2 n, n$ a positive integer, (b) $V(G)$ is the disjoint union of two sets each inducing a cycle $C$ of order $n$, and (c) $G$ is the total graph of $C$.

(ii) Let $v$ be a vertex of a graph $H$, and let $G$ be a maximal complete graph contained in the subgraph induced by $\bar{N}(v)$. Then $H$ $=T(K)$ if and only if $H=T(G)$.

The procedure given in the proof of Theorem 2 for determining whether or not a connected graph $H, H \neq T(C), T(K)$, is total seems rather long. There are of ten easy ways to fix $v$ or to eliminate many possibilities for $u_{1}$ and $v_{1}$. For example, if $H$ has odd vertices, then $v$ might be taken to be an odd vertex of $H$.

The following theorem is useful for the determination of those graphs which are total graphs of connected graphs having vertices of degree 1.

THEOREM 4. Let $H$ be a connected nonregular total graph with a vertex $v_{1}$ of degree 2 adjacent with vertices $v$ and $u_{1}$ of $H$. Then:

(a) $\operatorname{deg} u_{1} \neq \operatorname{deg} v$, say $\operatorname{deg} v<\operatorname{deg} u_{1}$;

(b) $v$ is nonspecial while $u_{1}$ and $v_{1}$ are the special vertices of $H$ having the property that $v$ corresponds, under the total graph function, to the edge $u_{1} v_{1}$.

Proof. It is clear that the degree of each nonspecial vertex of $H$ is greater than two. Thus $v_{1}$ is special. Hence either $u_{1}$ or $v$ is nonspecial. Assume deg $u_{1}=\operatorname{deg} v$. Then $\operatorname{deg} u_{1}=\frac{1}{2} \operatorname{deg} v+1$, or $\operatorname{deg} v=\frac{1}{2} \operatorname{deg} u_{1}+1$, both implying that $H$ is regular. Thus we may assume that deg $v$ $<\operatorname{deg} u_{1}$.

To prove part (b) suppose that $u_{1}$ is nonspecial. Then deg $u_{1}=$ $\frac{1}{2} \operatorname{deg} v+1$ and the inequality $\operatorname{deg} v<\operatorname{deg} u_{1}$ implies the impossible in- 
equality $\operatorname{deg} v_{1} \leqq 1$. Hence $v$ is nonspecial and clearly $v$ corresponds to the edge $u_{1} v_{1}$.

Theorems 3 and 4 provide an easy and practical characterization of total graphs of trees.

\section{REFERENCES}

1. M. Behzad, $A$ criterion for the planarity of the total graph of a graph, Proc. Cambridge Philos. Soc. 63 (1967), 679-681. MR 35 \#2771. 181.

2. - The connectivity of total graphs, Australian Math. Bull. 1 (1969), 1753.

3. - The total chromatic number of a graph: A survey, Proc. Conference Combinatorial Mathematics (Oxford, England, 1969), Academic Press, New York, 1970.

4. M. Behzad and G. Chartrand, Total graphs and traversability, Proc. Edinburgh Math. Soc. (2) 15 (1966/67), 117-120. MR $36 \# 1351$.

5. - An introduction to theory of graphs, Allyn and Bacon, Boston, Mass. (to appear).

6. M. Behzad and H. Radjavi, The total group of a graph, Proc. Amer. Math. Soc. 19 (1968), 158-163. MR 36 \#1358.

7. - Structure of regular total graphs, J. London Math. Soc. 44 (1969), 433-436. MR 38 \#4344.

8. F. Harary, Graph theory, Addison-Wesley, Reading, Mass., 1969.

Pahlavi University, Shiraz, Iran

Institute for Mathematical Research, The Imperial Scientific Research Organization, Tehran, Iran 